\title{
RÉGIONS ET VILLES SOCIALEMENT CRÉATIVES. ÉTUDE APPLIQUÉE À LA PÉNINSULE IBÉRIQUE
}

Isabel André et André Carmo

De Boeck Supérieur | «Innovations »

2010/3 n 33 | pages 65 à 84

ISSN 1267-4982

ISBN 9782804161545

Article disponible en ligne à l'adresse :

https://www.cairn.info/revue-innovations-2010-3-page-65.htm

Distribution électronique Cairn.info pour De Boeck Supérieur.

(C) De Boeck Supérieur. Tous droits réservés pour tous pays.

La reproduction ou représentation de cet article, notamment par photocopie, n'est autorisée que dans les limites des conditions générales d'utilisation du site ou, le cas échéant, des conditions générales de la licence souscrite par votre établissement. Toute autre reproduction ou représentation, en tout ou partie, sous quelque forme et de quelque manière que ce soit, est interdite sauf accord préalable et écrit de l'éditeur, en dehors des cas prévus par la législation en vigueur en France. Il est précisé que son stockage dans une base de données est également interdit. 


\section{RÉGIONS ET VILLES SOCIALEMENT CRÉATIVES. ÉTUDE APPLIQUÉE À LA PÉNINSULE IBÉRIQUE}

Isabel ANDRÉ Institut de Géographie et Aménagement du Territoire, Université de Lisbonne isabelandre@campus.ul.pt

André CARMO

Institut de Géographie et Aménagement du Territoire, Université de Lisbonne carmo@campus.ul.pt

Dans les sociétés postindustrielles ou postmodernes, la créativité - associée souvent à la culture et aux arts - est entendue comme un facteur essentiel du développement régional et urbain. Afin de promouvoir l'innovation et sa diffusion, les régions et les villes essaient depuis quelques années de favoriser la créativité. Très souvent cette politique se base sur l'attraction de personnes créatives (Landry, 2000 ; Florida, 2002) qui sont considérées comme une contribution importante pour la constitution d'un milieu « effervescent » et potentiellement créatif (Bourdin, 2010).

Pour définir l'essentiel de la ville créative, Florida (2002) met en relief les «3T » : Technologies, Talents et Tolérance. La première condition est associée à l'innovation technologique, la deuxième à l'éducation et au savoir, la troisième à la faculté de faire différemment. Nuur et Laestadius (2006, p. 6) font référence à Andersson et Strömquist, qui, dès 1988, avaient déjà associé le développement des territoires à des conditions similaires nommées les « $4 \mathrm{~K} »$ : Kunskap (Connaissances), Konst (Art), Kreativitet (Créativité) et Kommunikationer (Communication).

L'association des conditions économiques, sociales et culturelles dans une vision intégrée des stratégies urbaines est intéressante et avantageuse du point de vue du développement des villes. D'un autre côté, il est aussi important de souligner que le débat sur la ville créative présente les personnes (les 
talents) en tant que protagonistes principaux, mettant en évidence son rôle dans le développement économique et ces choix concernant les espaces et les activités urbaines. Les visions traditionnelles ont attribué cette responsabilité aux institutions et surtout aux entreprises.

En tout cas, malgré l'image de succès liée à la ville créative, ce modèle de développement urbain comporte des problèmes complexes du point de vue spatial et social : (i) la diversité des valeurs, attitudes et pratiques des personnes qui sont étiquetées comme classe créative et qui en réalité, forment des groupes assez distincts, notamment en ce qui concerne leurs valeurs et attitudes, ainsi que leurs relations avec la ville ; (ii) la fragmentation spatiale la création d'îles créatives isolées des autres espaces urbains ; (iii) l'exclusion sociale associée à l'éloignement des populations qui résidaient auparavant dans les espaces qui font l'objet de l'opération urbaine. En réalité, la ville créative n'est pas, dans bien des cas, une ville inclusive, mais plutôt un territoire qui prétend améliorer son image pour être mieux placé dans le ranking de la compétitivité nationale ou internationale.

Selon notre avis, on peut étendre le concept de milieu créatif comme celui qui favorise le changement culturel, encouragent la création et sa transformation en de nouvelles connaissances et qui est aussi capable de diffuser l'innovation technologique et sociale (Liefooghe, 2010). Il s'agit d'un milieu qui préserve sa cohérence et identité ; c'est-à-dire un milieu plastique, où les impacts du changement ne provoquent pas de fragmentation. Pour concrétiser notre débat sur la diversité de la " classe » créative et aussi sur le concept de milieu créatif (socialement créatif) nous avons analysé les régions et les villes de la Péninsule Ibérique. Il s'agit de territoires avec des niveaux de développement très différents et des représentations bien contrastées en termes de créativité et innovation. Utilisant les données de l'European Social Survey de 2006, nous avons réalisé une typologie de ces territoires qui nous permet de développer la matrice conceptuelle et méthodologique, associant diversité socioculturelle, tolérance, démocratie et capital relationnel, conditions que nous estimons cruciales pour définir un milieu socialement créatif.

Cet article discute l'importance de la créativité et de l'innovation dans le développement des territoires. On identifie là quelques «zones d'ombre », liées à un concept imprécis de classe et une idée restreinte de créativité qui laisse de côté l'innovation sociale, les stratégies socialement créatives associées à la connaissance tacite, à l'identité collective des communautés et à la participation civique. L'analyse des régions et des villes de l'Espagne et du Portugal illustre ce débat et nous conduit à la fois à d'autres questions. Les villes « sèchent-elles » les régions qui les entourent attirant presque tous les 
créatifs ou, bien au contraire, fonctionnent-elles comme images et leviers des espaces régionaux ? Quelle est la relation entre les composants économiques, sociaux et culturels des territoires créatifs? Quelle est l'échelle et le contexte le plus approprié pour développer un milieu socialement créatif ?

\section{CRÉATIVITÉ ET INNOVATION DANS LE DÉVELOPPEMENT DES TERRITOIRES}

On pourrait être tenté de dire que la créativité est une condition pour l'innovation, dans la mesure où elle fait émerger des idées originales, de nouvelles réponses et de nouveaux enjeux. (Runco, 2004). Cependant, ces deux termes recouvrent des significations différentes dans la littérature des sciences sociales. La créativité est ancrée dans la culture et dans les arts, restant très liée à l'imagination. L'innovation est surtout associée à la science et à la technique, bien que dans la dernière décennie se soit développé un domaine important dans les sciences sociales relatif à l'innovation sociale, associée à l'économie sociale et à la responsabilité sociale des entreprises ainsi qu'au rôle des communautés locales dans la résolution collective de leurs problèmes (Klein, Harrisson, 2007 ; Moulaert et al., 2009).

Dans un contexte d'incertitude et d'instabilité croissante, la promotion de la créativité est fondamentale dans la mesure où la pensée rationnelle et la logique du step-by-step, liées à l'innovation technologique, empêchent l'expression de l'imagination et la possibilité de trouver des réponses satisfaisantes. C'est la pensée divergente (Guilford, 1967) ou latérale (De Bono, 2008) - les utopies éventuellement réalisables - qui permettent de tracer de nouveaux parcours. D'ailleurs, une vision intégrée de la réalité sociale est indispensable pour trouver des solutions appropriées. Les problèmes économiques ne peuvent pas être uniquement résolus avec le savoir de l'économie, de même pour les problèmes concernant la santé, qui ne se résolvent pas uniquement avec la médecine. Les rapports sociaux, les valeurs morales, le cadre culturel, le contexte géographique ou les tensions politiques sont des facteurs décisifs pour arriver à comprendre les réalités et les situations plus spécifiques (Mouchiroud, Bernoussi, 2008). Le concept de créativité nous donne une vision intégrée et intégrante de la réalité, où toutes les solutions sont mises en équation mêmes celles qui ne sont pas applicables tout de suite. Ce sont ces caractéristiques de la créativité qui en font un atout majeur dans le développement des entreprises, des villes ou des régions (Liefooghe, 2009). L'originalité peut jouer un rôle clé dans le cadre de la compétitivité des entreprises, des communautés et des territoires. Mais elle est également un facteur décisif de cohésion sociale et spatiale, des domaines où des défis de 
plus en plus complexes font le jour exigeant des réponses intégrées et originales.

En effet, la créativité est capable de dévoiler le trait distinctif des lieux. Depuis les années soixante, Jane Jacobs a développé une réflexion intéressante sur l'importance de la différence entre les villes, soulignant le rôle de l'esthétique et du spectacle dans la vie urbaine (Jacobs, Fincher, 1998). Pour leur part, les travaux de Richard Florida $(2002,2005,2008)$ et de Charles Landry $(2000,2006)$ introduisent et déroulent le concept de ville créative ou de territoire créatif, très souvent considéré comme un facteur clé des stratégies urbaines et des politiques locales.

Tout en incluant des dimensions telles que la tolérance face aux comportements sociaux alternatifs ou la participation civique, le concept de ville créative sert fréquemment à justifier la valorisation économique des espaces urbains par le biais de processus de gentrification à forte implication au niveau de l'exclusion sociale (Moulaert et al., 2004 ; Ley, 2003 ; Scott, 2006). C'est dans le cadre d'une vision critique, mais constructive, de la ville créative qu'émerge le concept de ville socialement créative (Gertler, 2004 ; André, Abreu, 2009) que nous aborderons ci-dessous, après une brève discussion sur la signification de l'emploi créatif.

\section{LES CRÉATIFS}

Selon Florida (2002), la classe créative est la nouvelle force motrice de la société et, en particulier, du développement urbain ; elle comprend ceux qui créent de nouvelles significations et aussi ceux qui sont engagés dans la résolution des nouveaux problèmes mobilisant la connaissance scientifique. Cette vision, particulièrement attractive pour le discours politique des autorités urbaines, génère dans le monde académique des réactions complètement opposées. Pour les uns, Florida et ses collègues ont réussi à présenter finalement une théorie intégrée de la ville postmoderne. Pour les autres, il y a de grandes ambigüités liées aux notions de classe créative et de ville créative qui cachent une lecture néolibérale renouvelée (Peck, 2005 ; Scott, 2006). Parmi les nombreuses positions divergentes, il nous semble possible de trouver de bonnes pistes et intuitions dans les deux camps.

Il y a, à notre avis, deux idées clés dans ce débat. La première concerne le rôle privilégié des stratégies individuelles et des communautés urbaines visà-vis des stratégies entrepreneuriales. Landry $(2000,2006)$ et Florida (2002, 2005,2008 ) soulignent le fait que l'innovation dépend plus des personnes (individuellement ou collectivement) que des organisations et que, parallèlement, les employeurs sont disposés à suivre ceux qui produisent l'innova- 
tion. Comme le soulignent Clifton et Cooke (2007), la recherche de Florida met en évidence la nécessité d'une vision élargie de l'attractivité des lieux incluant l'offre culturelle et pas seulement l'environnement naturel et bâti. La deuxième idée est celle de faire des artistes des acteurs principaux de la ville créative, incluant les moins favorisés qui sont liés à l'art public et aux arts de rue. Il s'agit bien sûr d'un argument économique mais qui peut avoir de forts impacts sociaux - les artistes lancent les semis de la créativité dans la ville et en même temps développent des activités qui attirent les autres créatifs (ingénieurs, économistes, académiques, etc.). Comme le souligne Ann Markusen (2006), beaucoup d'artistes participent activement à la vie politique, surtout dans les campagnes électorales où ils utilisent ses outils de communication privilégiés. Ils sont généralement une source d'opposition au statu quo social, appuyant ainsi souvent les partis de gauche. La perspective mainstream n'aborde pas ces thèmes, mais elle prend en compte l'ouverture sociale de la ville créative, soulignant l'importance de la présence de différents groupes ethniques et styles de vie distincts (Gertler et al, 2002).

Les critiques adressées à Florida et à ses émules sont très diverses et souvent très fortes. Il faut dire que la notoriété de ses livres et l'aspect spectaculaire de ses conférences sont perçus a priori négativement dans le monde académique qui est très réactif face aux «blockbusters » de la recherche scientifique. Néanmoins, il y a des réflexions critiques assez intéressantes et bien fondées. Le géographe Jamie Peck (2005) présente une des visions critiques les mieux argumentées. Il commence par dire qu'il y a beaucoup de "politically ambivalent arguments contained in The Rise of the Creative Class, which mixes cosmopolitan elitism and pop universalism, hedonism and responsibility, cultural radicalism and economic conservatism, casual and causal inference, and social libertarianism and business realism" (Peck, 2005, p. 741).

La critique essentielle concerne le concept de classe. On ne peut pas trouver une base politique ou sociale, c'est-à-dire des intérêts collectifs, pour cette classe. Défini comme des personnes "... who seem to be always working, and yet never working when they are supposed to" (Florida, 2002, p. 5), ce groupe présente des caractéristiques spécifiques presque toutes liées au niveau d'éducation scolaire (Peck, 2005 ; Markussen, 2006 ; Boschma, Fritsch, 2007) et à son origine sociale bourgeoise.

Dans un autre vocabulaire, plus journalistique, la classe créative de Florida correspond aux «bobos » (bohèmes et bourgeois) dépeints par Brooks (2000). Brooks dit que jusqu'aux années 1960, la bourgeoisie adoptait un comportement austère (ou avait au moins une façade austère) complètement imperméable à l'aspect bohème qui caractérisait les classes populaires ou celles qui reniaient leur origine bourgeoise. Toutefois, Brooks fait remarquer 
qu'on a assisté, durant les dernières décennies, au déclin de l'austérité bourgeoise et à l'adoption d'attitudes guidées par le plaisir, par l'ostentatoire et par le spectacle. En effet, il s'agit peut-être d'une identité culturelle plus que d'une appartenance de classe. D'autre part, c'est un groupe avec une mobilité géographique élevée qui ne s'inscrit pas réellement dans les communautés urbaines où il peut vivre pendant une période limitée. Dans la recherche menée par Florida émerge même un esprit contraire au concept de classe sociale : l'illusion selon laquelle nous pouvons tous être créatifs - avantage du capital créatif par rapport au capital humain - et que nous pouvons tous jouir de la ville créative en faisant disparaître toute différence de classe.

Niant également le concept de classe créative comme unité sociologique, les recherches empiriques qui ont été réalisées ne confirment pas que les artistes attirent les business creatives. En réalité, ces business creatives consomment beaucoup de produits culturels, tangibles ou intangibles, mais ont un mode de vie très différent de celui des artistes. Ils n'ont pas besoin d'être proches les uns des autres au point de se déranger mutuellement, dès lors qu'ils ne partagent pas (ou plus) les mêmes valeurs et qu'ils ne développent pas des pratiques sociales semblables (Markusen, 2006).

\section{VILLES CRÉATIVES VERSUS MILIEUX SOCIALEMENT CRÉATIFS}

Dans le sillage de l'idée de ville créative, les autorités publiques mettent en œuvre toutes les stratégies pour attirer des personnes créatives ainsi que des investissements et des activités créatives. Cela se traduit par le financement de mégaprojets urbains liés non seulement à la culture et aux arts mais aussi aux infrastructures qui font fonctionner la ville créative (transports, télécommunications, réhabilitation de l'environnement, etc.). Néanmoins, le modèle «ville créative » a produit, dans les deux dernières décennies, des processus d'exclusion sociale assez problématiques, liés surtout à la gentrification et à la fragmentation socio-territoriale (Scott, 2006 ; Gertler, 2004 ; Miles, 1997). David Ley (2003) discute aussi à ce propos de l'appropriation du capital cultural collectif produit dans la ville par le marché, surtout par le marché immobilier.

Les projets créatifs de régénération urbaine se multiplient, perdant éventuellement la valeur attribuée par l'originalité (dimension cruciale de la créativité), manquant fréquemment de respect pour le cadre juridique de l'aménagement urbain et, surtout, de la volonté des populations qui se voient à maintes reprises éloignées des quartiers où elles ont engendré leurs relations sociales. Cette situation bien décrite par Moulaert et autres (2004) 
à propos de Bruges - Capitale Européenne de la Culture 2002 - correspond à la fragmentation de la ville avec le distancement croissant entre les zones chic, effervescentes et créatives - où se concentre la grande offre culturelle, alliée à l'offre commerciale - et les territoires périurbains de plus en plus déqualifiés (Markussen, 2006).

La régénération de la ville apparaît dans le discours politique comme une nécessité et un avantage, indépendamment des communautés affectées par ces processus. L'absence de préoccupation envers les impacts sociaux de la ville créative va plus loin. Il y a un ton de célébration de la classe créative qui laisse dans l'ombre le travail de milliers de travailleurs précaires considérés comme «non créatifs » - par contraste avec les « créatifs » / «bobos » qui garantissent une belle ville, propre et vibrante, prête à fonctionner 24 heures / 24, 7 jours / 7. Il est important de souligner que les arguments habituels sur la ville créative sont fréquemment transformés en discours facile et inconséquent par les autorités urbaines, comme un low-cost, market-friendly urban placebo (Peck, 2005, p. 760). Cela correspond, selon l'opinion de Peck, à l'érosion de la planification urbaine par le biais de la juxtaposition - incohérente et désintégrée - de projets immobiliers basés sur le pouvoir du marketing urbain. Mais les risques vont plus loin, s'associant au caractère éphémère des espaces créatifs, étroitement lié à l'impact des modes et à l'importance de la nouveauté, ce qui fait que ces espaces émergent et disparaissent facilement (Scott, 2006). Dans la même ligne de pensée, Scott attire l'attention sur l'importance de se promouvoir le creative-field effect lié à la communauté locale et à l'intensité des relations de proximité.

Ces visions critiques de la ville créative se concentrent sur deux points clés : la cohésion/fragmentation et la longévité/nature éphémère de la ville créative. D'un côté, la « classe créative » tend à se concentrer dans certains quartiers de la ville ou même dans de petites villes ayant de bons accès aux grandes métropoles et, par voie directe (projet de régénération urbaine) ou par voie indirecte (à travers l'augmentation du prix des maisons), finissant par éloigner une grande partie de la population qui, effectivement, ne partage pas ni ne jouit des avantages des milieux - physiques et sociaux - créatifs. De l'autre, les «super-créatifs » liées au business core ont une mobilité considérable, n'arrivant pas, souvent, à créer des racines et à laisser des graines dans les endroits où ils se sont installés.

Mais, malgré les menaces référées aux paragraphes précédents, nous estimons que la présence d'artistes en ville, l'art public, les nouveaux landmarks culturels ou le street art peuvent encourager d'une manière significative l'auto-estime des communautés locales et régionales et renforcent, de ce fait, le sentiment d'appartenance territoriale, particulièrement important dans des villes multiethniques où l'on a constamment une population qui va et 
vient. Ces potentialités pourront être accrues si les artistes et les activités qui leur sont associées sont amenés à s'installer dans des zones plus problématiques, où le risque de fragmentation spatiale et d'exclusion sociale sont plus importants. C'est dans ces endroits que l'esthétisation des espaces, ainsi que la communication et le dialogue à travers les arts favorisent le plus le développement social et urbain. Toutefois, ce n'est pas cela qui se passe en général. Dans la grande majorité des cas, les villes créatives ne sont pas des villes inclusives ni innovatrices du point de vue social. Aussi, les discours alternatifs sur la ville créative deviennent-ils chaque jour plus puissants, introduisant le concept de ville socialement créative, celle qui encourage la créativité favorisant en même temps la cohésion (Gertler, 2004 ; Scott, 2006).

Les autorités publiques du Canada, et de Montréal en particulier, semblent être attentives au débat critique sur la ville créative et aux visions alternatives (Tremblay, Klein, Fontan, 2005). Gertler (2004, p. 1) souligne que « l'objectif de la politique publique au Canada devrait être - et peut être - de renforcer la formation des lieux créatifs socialement inclusives ». Cette ambition des politiques urbaines canadiennes montre qu'il est possible de penser et construire des lieux où la créativité inspire non seulement l'innovation technologique mais aussi l'innovation sociale. Cela signifie combiner d'une part le dynamisme économique et le changement par anticipation et, d'autre part, la transformation des relations sociales et la production de capital social collectif surtout dans les communautés les plus fragiles (Klein et Harrison, 2007 ; Moulaert et al., 2009). Il faut donc identifier quelles sont les dynamiques à mettre en œuvre dans ces endroits. Qu'est-ce qui fait d'eux des milieux socialement créatifs? À notre avis, le milieu socialement créatif est celui où le changement culturel consistant est possible sans perdre sa cohérence et identité en tant que lieu. Le milieu qui favorise la créativité permet la transformation de la création en nouvelles connaissances ou visions, et diffuse l'innovation technologique et sociale. Il s'agit d'un milieu plastique, où les impacts du changement ne provoquent pas de fragmentation.

La notion de plasticité (adoptée de la physique) - la capacité de déformation et en formation (recevoir une nouvelle forme) sans perdre l'unité et la cohérence - paraît être une idée utile pour définir ces milieux. Ce concept «peut bien illustrer la nature spécifique des milieux créatifs. Ceux-ci devront être suffisamment flexibles et en même temps suffisamment organisés pour qu'ils puissent subir des transformations sans perdre leur identité » (André, Abreu, 2006, p. 132). En transférant le concept aux sciences sociales, les milieux socialement créatifs sont des territoires dynamiques qui préservent ou recréent leur identité. Cette capacité semble être le résultat de quatre conditions principales : 
- Diversité (valorisant la différence et l'altérité) - le nouveau vient de l'autre.

- Tolérance (permettant et même promouvant la prise de risque) - le nouveau est risqué.

- Démocratie (encourageant la mobilisation et la coopération) - le nouveau devient du dialogue.

- Capital relationnel collectif ou individuel mis au service des communautés locales, urbaines ou régionales - le nouveau devient de l'interaction.

La diversité renforce l'interaction et le contact avec le nouveau, le sens de l'altérité : nouveaux-autres produits, nouveaux-autres savoirs, nouvellesautres valeurs. La tolérance est la condition nécessaire du risque toujours associé à la création et à l'innovation (comme au trapèze, le risque suppose un filet). Un milieu ne peut être créatif s'il pénalise les éventuels insuccès d'une initiative risquée, c'est-à-dire si c'est trop hiérarchisé, normatif ou rigide. La démocratie implique la participation active, c'est-à-dire la possibilité et la capacité de décision, l'accès à l'information et à la connaissance nécessaires au choix et à l'identification des solutions et des réponses nouvelles et appropriées. Le capital relationnel fait référence à l'interaction qui provient soit des liens de proximité qui actionnent des réseaux locaux (de voisinage, familiaux, amicaux, etc.), soit de l'établissement de ponts vers l'extérieur. Il ne s'agit pas seulement du capital social des élites, mais surtout de la possibilité et de la capacité, collectivement appropriées, de rendre les territoires plus cohésifs et plus liés au reste du monde.

\section{LES GENS ET LES TERRITOIRES CRÉATIFS : LE CAS DE LA PÉNINSULE IBÉRIQUE}

La géographie de l'emploi créatif dans les villes et régions ${ }^{1}$ de la Péninsule Ibérique (Carte 1) - considérant les professions créatives, ainsi que l'emploi dans les activités créatives - permet de développer certains points du débat présenté avant.

1. La valeur de la ville (ou villes) a été soustraite au total de la région pour obtenir la situation du territoire sans l'effet des grandes villes. 
Carte 1 - Régions et villes (*) de l'Espagne et du Portugal

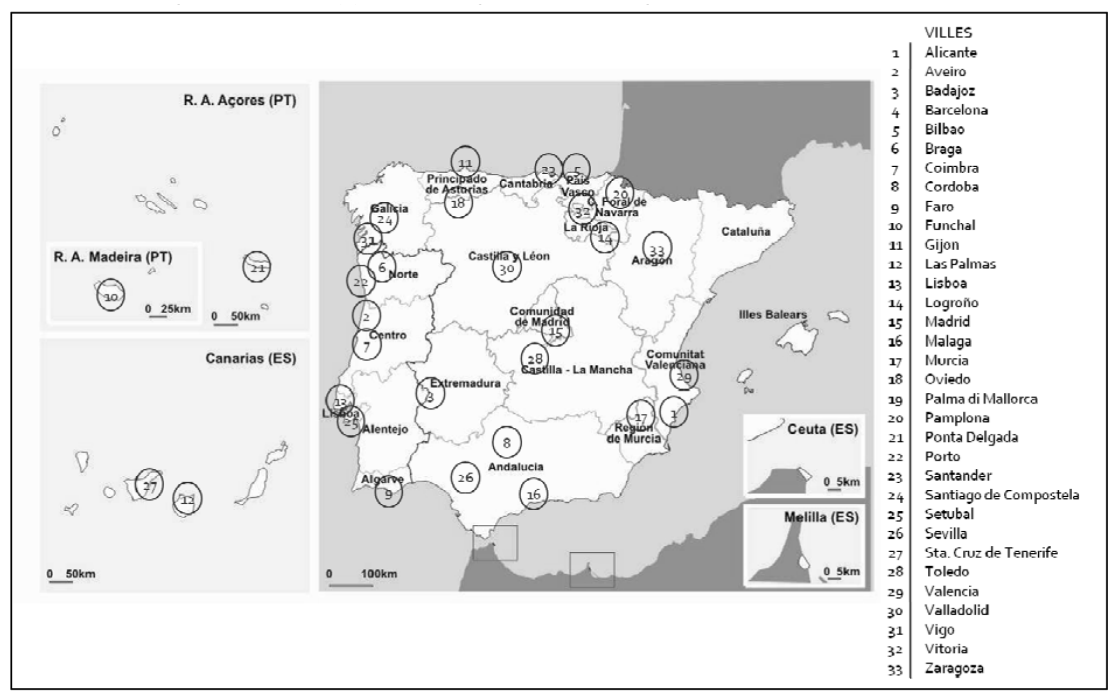

(*) Considérées par Urban Audit, Politique Régionale - Inforegio, Commission Européenne.

Comme nous l'avons suggéré dans le point précédent, nous considérons que les professions créatives - le Super Creative Core (SCC) défini par Florida (2002) - forment deux groupes distincts : i) les artistes; ii) les autres « super-créatifs ». Le premier segment rassemble les designers, les écrivains, les musiciens, les photographes, les cinéastes, les danseurs, les comédiens, les peintres, les sculpteurs, entre autres - qui engendrent de nouveaux sens et significations. Le second regroupe les scientifiques, les architectes, les ingénieurs, les professeurs universitaires, les opinion-makers et d'autres catégories similaires de professionnels - ceux qui produisent ou mobilisent des connaissances pour résoudre de nouveaux problèmes.

Malgré le fait que la centralité occupée par la créativité soit le trait distinctif du SCC, on considère que cette distinction marque une subtile mais importante différence qualitative entre les deux segments. Sans vouloir trop faire l'apologie de l'« Art » per se en tant que facteur crucial du développement des territoires, on admet que le premier segment est généralement marqué par une plus faible institutionnalisation, un plus grand sens critique et une plus forte liaison à la communauté locale et à son capital symbolique, tandis que, dans le second, le procès créatif est soumis à des logiques institutionnelles plus rigides, moins malléables (la créativité scientifique, par exemple, obéit à des règles processuelles et méthodologiques relativement strictes) et la mobilité résidentielle est plus accentuée, ce qui affaiblit les 
relations de proximité et l'identification avec les lieux. Cette division nous permet, selon notre ligne de pensée, d'appréhender l'hétérogénéité interne du super-creative core d'une manière qui ne serait pas possible si on la considérait comme une catégorie unique et homogène.

Dans un deuxième temps, nous allons analyser les activités créatives, en termes d'emploi, sachant que celles-ci recouvrent également deux segments : i) les activités culturelles et artistiques ; ii) les activités de recherche et développement $(R \& D)$. Ici, les différences entre les segments sont plus évidentes puisque le premier se place autour de la sphère d'influence des humanités, des lettres et de la culture, tandis que le second segment est immédiatement associé aux activités à caractère éminemment scientifique et technique, particulièrement importantes dans la société de la connaissance, dans laquelle la rationalité est le trait dominant, et l'innovation technologique un des principaux objectifs.

Le Graphique 1 (représentant le pourcentage des artistes face au pourcentage des autres 'super-créatifs dans l'emploi total de la ville ou région ${ }^{2}$ ) nous permet de vérifier que, en général, la géographie du SCC se caractérise essentiellement par ce que nous pouvons nommer l'« effet-agglomération », c'est-à-dire par une concentration des créatifs dans les villes, en particulier dans les métropoles/villes capitales, comme Madrid, Lisbonne et Barcelone. Toutefois, on souligne également l'importance de certaines villes de taille moyenne comme Saint-Jacques de Compostelle, Bilbao, Porto, Valence, Oviedo et Séville. Cet ensemble de villes se divise lui-même en deux sousgroupes : l'un où prédominent, en termes relatifs, les artistes face aux autres "super-créatifs » (Madrid, Lisbonne, Barcelone et Saint-Jacques de Compostelle), et l'autre où ces artistes sont relativement moins représentés. Le cas extrême de cette seconde situation est Coimbra, où le pourcentage d'artistes est assez inférieur à la moyenne et où le poids relatif des autres « super-créatifs » est le plus élevée dans la Péninsule Ibérique.

Le Graphique 2 (représentant le pourcentage de l'emploi aux activités culturelles et artistiques face au pourcentage de l'emploi aux activités de $R \& D^{3}$ ) vient confirmer les évidences décrites dans le paragraphe précédent. La situation probablement la plus intéressante du point de vue analytique est celle des territoires où les artistes/activités culturelles et artistiques sont audessus de la moyenne, et les autres super-créatifs/activités de R\&D sont en dessous de la moyenne.

2. Les données sur l'emploi proviennent des recensements de population de l'Espagne et du Portugal, 2001.

3. R\&D : Recherche \& Développement. 
Graphique 1 - Analyse bivariée de l'emploi créatif par segments professionnels

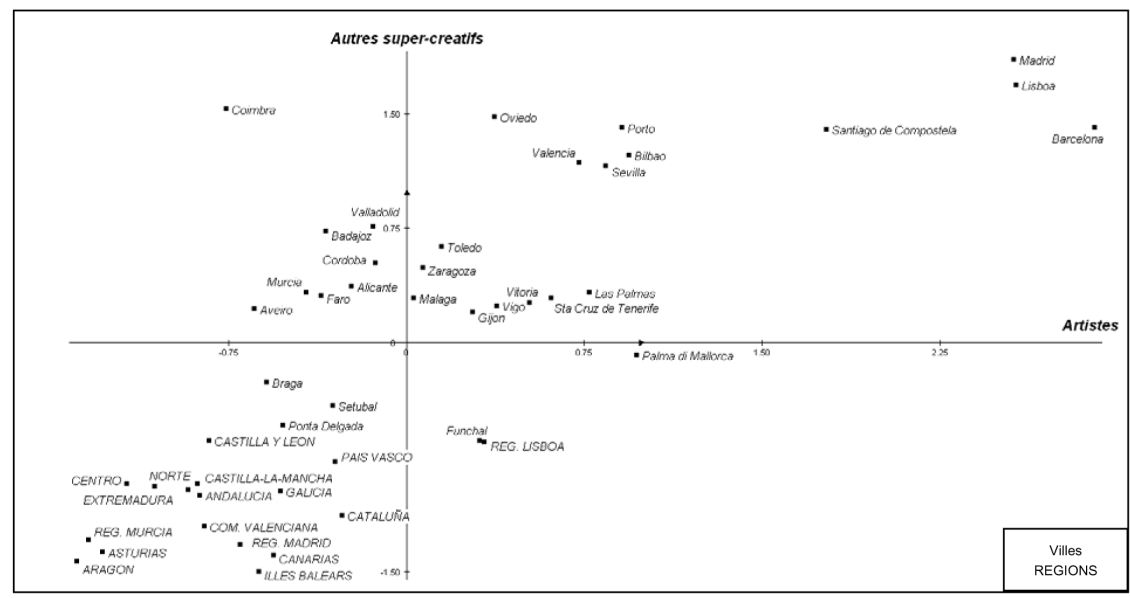

Graphique 2 - Analyse bivariée de l'emploi créatif par activités

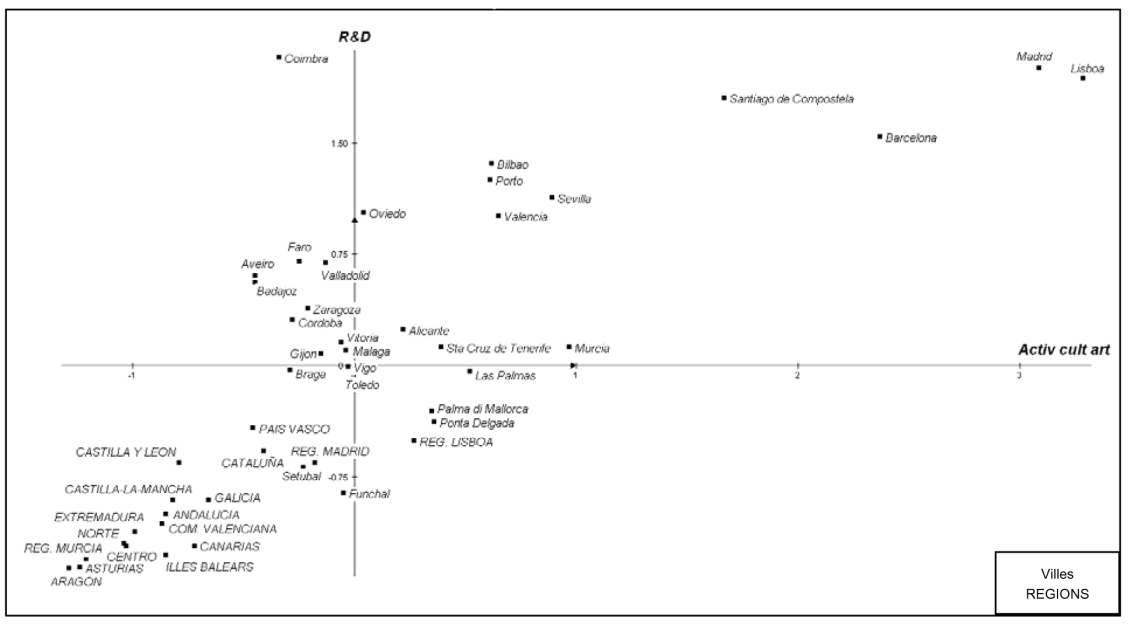

Curieusement, ceci se passe exclusivement avec des villes insulaires Palma de Mallorque, Las Palmas, Funchal et Ponta Delgada. Comment la situation insulaire peut-elle être liée à la surreprésentation des métiers et activités artistiques ? Elle doit vraisemblablement être liée au rôle important joué par le tourisme, mais également être associée à l'existence d'environnements qui « inspirent » du point de vue des paysages ou des modes de vie. Il est également important de souligner que les régions accompagnent leurs villes. C'est-à-dire, par exemple le Pays Basque (sans Bilbao) ou la Catalogne 
(sans Barcelone) montrent un plus grand pourcentage d'artistes et d'emplois culturels ou artistiques que les autres régions. Mais la situation la plus remarquable est celle de la Région de Lisbonne, qui se distingue clairement de toutes les autres régions par le fait qu'elle présente - même en isolant la ville capitale - une proportion d'artistes et d'emplois dans les activités culturelles et artistiques assez élevée. Ceci est certainement dû au poids de certaines villes périurbaines qui entourent la capitale - Cascais, Sintra, Oeiras, parmi d'autres - où les facteurs d'attraction sont importants (localisation sur la côte, équipements culturels, urbanisme de qualité, etc.).

Bref, la géographie de la créativité dans la Péninsule Ibérique fait ressortir clairement les plus grandes villes et, plus particulièrement, les villes capitales. Ceci démontre que la masse critique est un facteur important d'attraction de créatifs et particulièrement des artistes. L'autre facteur assez significatif est la présence d'universités ou d'autres centres de $R \& D^{4}$. Les valeurs notées à Saint-Jacques de Compostelle et à Coimbra illustrent bien cette relation.

\section{Les milieux socialement créatifs ont-ils la même géographie ?}

Les données produites par l'European Social Survey (2006) nous permettent de retenir un ensemble d'informations utiles sur les caractéristiques associables aux milieux socialement créatifs : diversité socioculturelle, tolérance, démocratie et capital relationnel. Le tableau 1 présente les indicateurs sélectionnés pour l'analyse ${ }^{5}$. En nous basant sur ces indicateurs, des indices synthétiques ont été construits résumant chacune des dimensions d'analyse.

4. Chantelot (2009) retrouve le même patron en France où l'expression de la classe créative est surtout intense à Paris et dans les villes de plus grande dimension, mais aussi dans des villes moyennes comme Lannion ou Vernon, dû à la présence de centres de R\&D.

5. Les données de l'ESS (http://ess.nsd.uib.no/) sont disponibles en nomenclature NUT2. Pour cette raison, l'analyse est effectuée uniquement au niveau régional et au sein de la Péninsule Ibérique (à l'exception de l'Algarve, de la Cantabria, de la Navarre et de la Rioja, pour lesquelles il n'a pas été possible de garantir la rigueur des indicateurs sélectionnés), et non pas au niveau des villes. 
Tableau 1 - Indicateurs de milieux socialement créatifs

\begin{tabular}{|c|c|}
\hline \multirow{4}{*}{ DIVERSITÉ } & Pourcentage de population appartenant à une minorité ethnique \\
\hline & Pourcentage de population dont la mère est née en dehors du pays \\
\hline & $\begin{array}{l}\text { Indice de diversité électorale (coefficient de variation du vote partisan aux élec- } \\
\text { tions législatives) }\end{array}$ \\
\hline & $\begin{array}{l}\text { Indice de diversité religieuse (coefficient de variation du \% d'adeptes de chaque } \\
\text { religion) }\end{array}$ \\
\hline \multirow{6}{*}{ TOLÉRANCE } & $\begin{array}{l}\text { Pourcentage de population considérant que gays et lesbiennes sont libres de } \\
\text { vivre leur vie comme ils/elles le souhaitent }\end{array}$ \\
\hline & $\begin{array}{l}\text { Pourcentage de population considérant que l'immigration est positive pour } \\
\text { l'économie nationale. }\end{array}$ \\
\hline & $\begin{array}{l}\text { Pourcentage de population considérant que la vie culturelle est enrichie via les } \\
\text { immigrés }\end{array}$ \\
\hline & $\begin{array}{l}\text { Pourcentage de population considérant que les immigrés font du pays une } \\
\text { meilleure place pour vivre }\end{array}$ \\
\hline & Pourcentage de population qui se sent libre pour décider comment vivre sa vie \\
\hline & $\begin{array}{l}\text { Pourcentage de population qui considère important qu'il y ait égalité de traite- } \\
\text { ment et d'opportunités }\end{array}$ \\
\hline \multirow{9}{*}{ DÉMOCRATIE } & Pourcentage de population intéressée à la politique \\
\hline & $\begin{array}{l}\text { Pourcentage de population qui a participé durant la dernière année à un parti } \\
\text { politique ou groupe d'action }\end{array}$ \\
\hline & $\begin{array}{l}\text { Pourcentage de population qui a participé durant la dernière année à une orga- } \\
\text { nisation ou association }\end{array}$ \\
\hline & Pourcentage de population qui a signé durant la dernière année une pétition \\
\hline & $\begin{array}{l}\text { Pourcentage de population qui durant la dernière année a participé à une } \\
\text { manifestation publique }\end{array}$ \\
\hline & $\begin{array}{l}\text { Pourcentage de population qui durant la dernière année a participé à un boy- } \\
\text { cott d'au moins un produit }\end{array}$ \\
\hline & Pourcentage de population qui durant la dernière année a fait du bénévolat \\
\hline & $\begin{array}{l}\text { Pourcentage de population qui durant la dernière année a aidé d'autres per- } \\
\text { sonnes (excepté la famille, les collègues ou le bénévolat) }\end{array}$ \\
\hline & $\begin{array}{l}\text { Pourcentage de population qui durant la dernière année a participé à des acti- } \\
\text { vités dans sa zone résidentielle }\end{array}$ \\
\hline \multirow{6}{*}{$\begin{array}{l}\text { CAPITAL } \\
\text { RELATIONNEL }\end{array}$} & Pourcentage de population qui utilise Internet et/ou le courriel \\
\hline & Pourcentage de population qui participe à des activités sociales \\
\hline & $\begin{array}{l}\text { Pourcentage de population qui s'est rarement ou jamais sentie seule durant la } \\
\text { semaine dernière }\end{array}$ \\
\hline & $\begin{array}{l}\text { Pourcentage de population qui considère que les personnes s'entraident dans } \\
\text { son lieu de résidence }\end{array}$ \\
\hline & $\begin{array}{l}\text { Pourcentage de population qui considère que les autres personnes s'intéres- } \\
\text { sent à elle }\end{array}$ \\
\hline & $\begin{array}{l}\text { Pourcentage de population qui se sent proche des autres personnes dans son } \\
\text { lieu de résidence }\end{array}$ \\
\hline
\end{tabular}


La corrélation entre les différentes catégories d'emplois créatifs, analysées antérieurement, et les indices synthétiques des milieux socialement créatifs (Tableau 2), montre qu'il n'y a pas de relations statistiquement significatives entre les deux ensembles. Ceci signifie que les régions où les créatifs ont la plus forte expression relative ne sont pas celles qui correspondent, selon nos critères, aux milieux socialement créatifs. Par contraste, l'association des créatifs au niveau d'éducation et au PIB per capita est très significative. Les régions les plus riches et à capital humain plus qualifié sont celles qui ont les pourcentages supérieures d'emplois créatifs.

Tableau 2 - Matrice des corrélations entre créatifs et dimensions analytiques des milieux socialement créatifs (base-NUT2 de la Péninsule Ibérique)

\begin{tabular}{|l|c|c|c|c|c|c|c|c|c|}
\hline & C2 & C3 & C4 & C5 & C6 & C7 & C8 & C9 & C10 \\
\hline C2 Artistes & 1.00 & & & & & & & & \\
\hline C3 Autres super créatifs & 0.63 & 1.00 & & & & & & & \\
\hline $\begin{array}{l}\text { C4 Activités culturelles. } \\
\text { artistiques }\end{array}$ & $\mathbf{0 . 9 1}$ & $\mathbf{0 . 8 1}$ & $\mathbf{1 . 0 0}$ & & & & & & \\
\hline C5 Activités R\&D & $\mathbf{0 . 8 5}$ & $\mathbf{0 . 9 0}$ & $\mathbf{0 . 9 0}$ & 1.00 & & & & & \\
\hline C6 Éducation universitaire & $\mathbf{1 . 0 0}$ & 0.63 & $\mathbf{0 . 9 1}$ & $\mathbf{0 . 8 5}$ & 1.00 & & & & \\
\hline C7 PIB/capita & $\mathbf{0 . 7 8}$ & $\mathbf{0 . 7 2}$ & $\mathbf{0 . 7 0}$ & $\mathbf{0 . 8 7}$ & $\mathbf{0 . 7 8}$ & 1.00 & & & \\
\hline C8 Indice Participation & 0.29 & 0.16 & 0.23 & 0.33 & 0.29 & 0.44 & 1.00 & & \\
\hline C9 Indice Diversité & 0.19 & 0.07 & 0.13 & 0.17 & 0.19 & 0.39 & 0.56 & 1.00 & \\
\hline C10 Indice Tolérance & 0.29 & -0.06 & 0.07 & 0.19 & 0.29 & 0.38 & $\mathbf{0 . 7 4}$ & 0.46 & 1.00 \\
\hline C11 Indice Cap. Relationnel & 0.12 & -0.28 & -0.11 & -0.04 & 0.12 & 0.21 & 0.52 & 0.34 & $\mathbf{0 . 8 6}$ \\
\hline
\end{tabular}

L'analyse multi variée (composantes principales) permet d'analyser plus en détail la relation entre ces indicateurs et de la projeter dans les unités d'analyse (régions). Les deux premiers facteurs expliquent $81 \%$ de la variance totale, associant, d'un côté, les segments d'emplois créatifs, la richesse et le niveau d'éducation ( $1^{\mathrm{er}}$ facteur), et de l'autre, les indices associés aux milieux socialement créatifs ( $2^{\mathrm{e}}$ facteur) (Graphique 3 ). Ce que cette procédure a de plus intéressant, c'est l'association des régions aux deux premiers facteurs (Graphique 4). 
Graphique 3 - Définition des facteurs - milieux créatifs

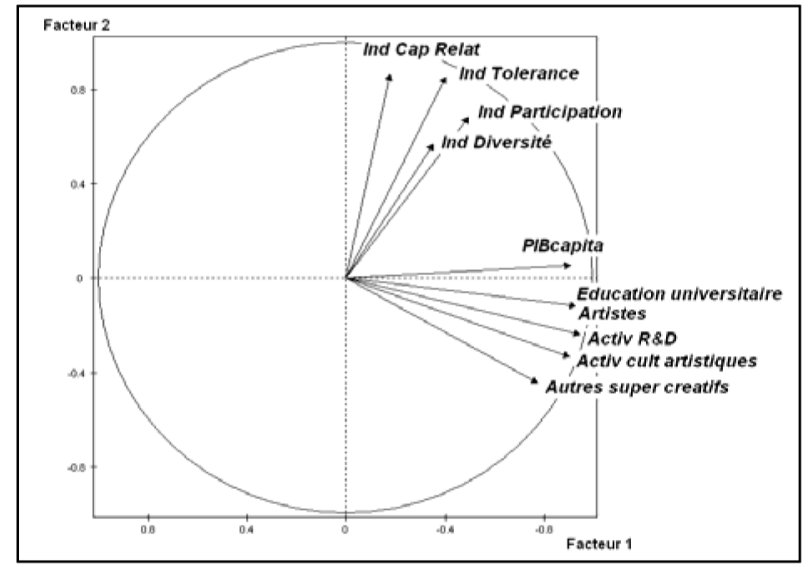

Graphique 4 - Projection des unités d'analyse dans les axes factoriels - milieux créatifs

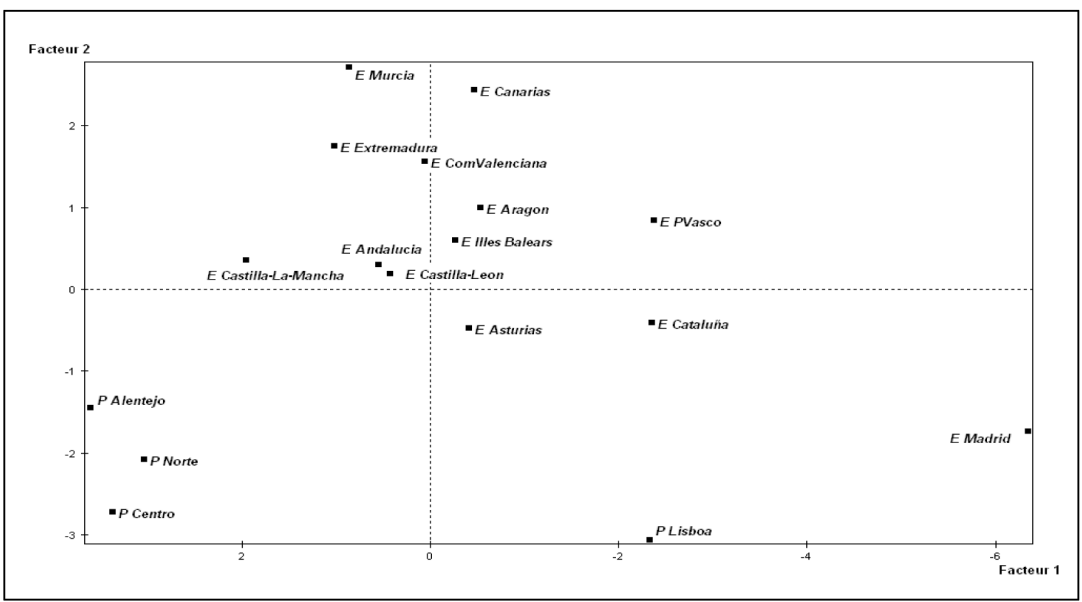

On définit 4 groupes de régions à partir de leur position dans les quadrants du graphique :

- Dans la situation la plus défavorable, il y a 3 régions portugaises avec une sous représentation d'emplois créatifs et aussi des conditions négatives qui configurent les milieux socialement créatifs.

- Également en situation négative face à l'axe 2 - milieux socialement créatifs - mais avec des valeurs très élevées dans l'axe 1 emplois créatifs - surgit de manière isolée la région de Madrid, accom- 
pagnée, à une certaine distance, par la Catalogne, Lisbonne et les Asturies.

- La plupart des régions espagnoles présente des valeurs positives dans l'axe 2 et une position négative dans l'axe 1.

- La situation des 4 autres régions espagnoles - le Pays Basque, l'Aragon et les Iles Canaries et Baléares - est assez intéressante, elles allient l'emploi créatif et les conditions favorables des milieux socialement créatifs.

Il s'agit d'une analyse exploratoire qui nécessite d'être consolidée sur la base d'informations complémentaires. Néanmoins, il en ressort déjà plusieurs pistes intéressantes pour la compréhension des liens entre les territoires créatifs définis dans l'optique du mainstream (à partir de l'emploi et des activités économiques) et les milieux socialement créatifs associés à la promotion de l'innovation sociale. Les données que nous avons analysées montrent que ce n'est pas dans les grandes métropoles que cette association s'observe. Les valeurs de Madrid, de Lisbonne et de la Catalogne révèlent que le mix diversité-démocratie-tolérance-capital relationnel n'y est pas très expressif.

\section{CONCLUSION}

La créativité implique une attitude proactive et une vision holistique de la réalité. Elle convoque l'imagination et les émotions, renforçant la capacité de faire face à l'incertitude et au changement rapide, aidant à confronter les tensions et les conflits. Dans le cas des territoires, la promotion de la créativité vise surtout la production du trait distinctif correspondant actuellement surtout à l'avantage compétitif dans la sphère économique. La culture et les arts jouent ici un rôle décisif, étant donné qu'ils aident à renforcer l'identité spécifique des lieux et des communautés, tandis que la science et la technologie sont de plus en plus globales, contribuant ainsi de moins en moins à la distinction des territoires, soit des villes ou des régions.

La littérature sur la classe et la ville créative, en particulier l'œuvre de Richard Florida, associe la créativité à l'innovation scientifique et technologique rassemblant tous les types de créatifs dans un même groupe social, considéré comme l'agent crucial de la ville créative. Cette vision n'est cependant pas exempte de plusieurs critiques importantes. En particulier, il faut souligner l'hétérogénéité de la classe créative, ce qui remet en cause le concept même de classe. Parallèlement, la conception réductrice de la ville créative, notamment au travers du prisme de la « régénération urbaine créative ", conduit fréquemment à des processus de fragmentation spatiale et 
d'exclusion sociale. Enfin, il faut mentionner que la narrative da la ville créative se transforme, à plusieurs reprises, en discours politique simpliste et attractif, mais qui ne prend en compte ni les modalités d'action, ni le changement social.

Répondant aux questions que nous avons formulées au début de l'article, la géographie ibérique de l'emploi créatif révèle quatre aspects qui méritent une attention particulière. Le premier est l'effet agglomération ou métropole, Madrid, Barcelone et Lisbonne présentant les plus hauts pourcentages d'artistes et des autres super-créatifs. Le second c'est l'effet de levier des métropoles sur les espaces régionaux où elles s'intègrent, particulièrement évident dans la région de Lisbonne. Le troisième est l'effet pole de connaissances : quelques villes où se trouvent des universités et des centres de recherche importantes de la Péninsule Ibérique comme Saint-Jacques de Compostelle ou Coimbra, ressortent de manière significative et en particulier, à travers la sur-représentation des autres super-créatifs. Le quatrième et le plus inattendu est l'effet insularité qui se rapporte au fort poids relatif des artistes. Effet du tourisme ? Du paysage? De la tranquillité ? Cette piste mérite certainement une réflexion future plus approfondie appuyée sur plus d'évidences.

Les villes font la promotion, à travers différentes stratégies, de leur créativité pour augmenter leur compétitivité, soit par la voie de l'innovation technologique, soit par l'impulsion du marché immobilier. Cela se produit souvent au détriment de la cohésion territoriale et sociale, et de l'innovation sociale, qui est bridée. Cette conviction fait émerger le concept de "milieu socialement créatif ». Spécialement à travers la diversité socioculturelle, la tolérance, la démocratie et la production de capital relationnel, la créativité peut promouvoir l'innovation sociale, rendant les lieux plus inclusifs à travers le renforcement de l'identité (ou la reconstruction des identités) et de l'auto-estime.

L'analyse de caractéristiques liées aux milieux socialement créatifs révèle qu'ils ne coïncident pas, dans la Péninsule Ibérique, avec les territoires où il y a une forte concentration d'emplois créatifs. Toutefois, surgissent des régions où l'emploi créatif s'associe aux attributs des milieux socialement créatifs. C'est le cas du Pays Basque, de l'Aragon et des Iles Canaries et Baléares. Cette conclusion relève de certaines questions pertinentes à savoir : quelle est l'échelle adéquate pour la constitution d'un milieu socialement créatif ? Les métropoles peuvent-elles être, dans son tout, des milieux socialement créatifs ? Ou seulement quelques quartiers? Les stratégies socialement créatives ont-elles plus de succès dans les villes de petite et moyenne dimension? Leur succès est-il dépendant de l'intensité des relations de proximité, de la consistance de la communauté locale ou dépend-il surtout des relations multi-échelles, du « glocal »? 


\section{RÉFÉRENCES BIBLIOGRAPHIQUES}

ANDRÉ, I., ABREU, A., 2006, Dimensões e espaços da inovação social, Finisterra, XLI (81), 121-141.

ANDRÉ, I., ABREU, A., 2009, Social creativity and post-rural places: the case of Montemoro-Novo, Portugal, Canadian Journal of Regional Science/Revue Canadienne des Sciences Régionales, Special issue on Social Innovation and Territorial Development, XXXII (1), 101-114.

BOURDIN, A., 2010, L'urbanisme d'après crise, La Tour d'Aigues, Paris, Éditions de l'Aube. BOSCHMA, R., FRITSCH, M., 2007, Creative Class and Regional Growth - Empirical Evidence from Eight European Countries, in Jacubowska, P. et al., The Future of European Regions, Warzaw, Ministry of Regional Development, 243-253.

BROOKS, D., 2000, Bobos in Paradise: the New Upper Class and How They Got There, New York, Simon \& Schuster.

CHANTELOT, S., 2009, La géographie de la classe créative : une application aux aires urbaines françaises, XLVI e colloque de l'ASRDLF - Entre projets locaux de développement et globalisation de l'économie : quels équilibres pour les espaces régionaux? 6-8 juillet, ClermontFerrand.

CLIFTON, N., COOKE, P., 2007, The 'Creative Class' in the UK: An Initial Analysis, Regional Industrial Research Report, Centre for Advanced Studies, Cardiff University.

DE BONO, E., 2008, Comment avoir des idées créatives, Paris, Leduc S. Editions.

FLORIDA, R., 2002, The Rise of the Creative Class: And How It's Transforming Work, Leisure, Community, and Everyday Life, New York, Basic Books.

FLORIDA, R., 2005, The Flight of the Creative Class: The New Global Competition for Talent, New York, Harper \& Collins.

FLORIDA, R., 2008, Who's Your City? How the Creative Economy Is Making Where to Live the Most Important Decision of Your Life, New York, Basic Books.

GERTLER, M., FLORIDA, R., GATES, G., VINODRAI, T., 2002, Competing on Creativity: Placing Ontario's Cities in North American Context, Report prepared for the Ontario Ministry of Enterprise, Opportunity and Innovation and the Institute for Competitiveness and Prosperity.

GERTLER, M., 2004, Creative Cities: What Are They For, How Do They Work, and How Do We Build Them?, Ottawa, Canadian Policy Research Networks.

GUILFORD, J. P., 1967, The Nature of Human Intelligence, New York, McGraw-Hill.

HALL, P., 2000, Creative Cities and Economic Development, Urban Studies, 37 (4), 639-649.

JACOBS, J., FINCHER, R., 1998, Cities of Difference, Nova Iorque, Guilford Press.

KLEIN J.-L., HARRISON D. (eds), 2007, L'innovation Sociale - Emergence et effets sur la transformation des sociétés, Québec, Presses de l'Université du Québec.

LANDRY, C., 2000, The Creative City: A Toolkit for Urban Innovators, London, Earthscan. LANDRY, C., 2006, The Art of City Making, London, Earthscan.

LIEFOOGHE, C., 2009, La créativité : une ressource pour le développement économique d'une région de tradition industrielle ?, XLVI colloque de l'ASRDLF - Entre projets locaux de développement et globalisation de l'économie : quels équilibres pour les espaces régionaux ? 6-8 juillet, Clermont-Ferrand. 
LIEFOOGHE, C., 2010, Economie créative et développement des territoires : enjeux et perspectives de recherche, Innovations. Cahiers d'économie de l'innovation, 31, 181-197.

LEY, D., 2003, Artists, Aestheticisation and the Field of Gentrification, Urban Studies, 40 (12), 2527-2544.

MARKUSEN, A., 2006, Urban development and the politics of a creative class: evidence from a study of artists, Environment and Planning A, 38 (10), 1921-1940.

MILES, M., 1997, Arts, Space and the City. Public Art and Urban Futures, London, Routledge.

MOUCHIROUD, C., BERNOUSSI, A., 2008, An empirical study of the construct validity of social creativity, Learning and Individual Differences, 18, 372-380.

MOULAERT, F., DEMUYNCK, H., NUSSBAUMER, J., 2004, Urban renaissance: from physical beautification to social empowerment. Lessons from Bruges - Cultural Capital of Europe 2002, City, 8 (2), 229-235.

MOULAERT, F., MACCALLUM, D., HILlIER, J., VICARI, S., 2009, Social innovation and Territorial Development, Farnham, Ashgate.

NUUR, C., LAESTADIUS, S., 2009, Is the 'Creative Class' Necessarily Urban? Putting the Creativity Thesis in the Context of Non-urbanised Regions in Industrialised Nations, Debate June 2009, European Journal of Spatial Development, http://www.nordregio.se/EJSD/ debate200906.pdf

PECK, J., 2005, Struggling with the creative class, International Journal of Urban and Regional Research, 29 (4), 740-770.

RUNCO, M. A., 2004, Creativity, Annual Review of Psychology, 55, 657-687.

SCOTT, A., 2006, Creative cities: Conceptual issues and policy questions, Journal of Urban Affairs, 28 (1), 1-17.

TREMBLAY, D.-G., KLEIN, J.-L., FONTAN, J.-M., 2005, Innovation socioterritoriale et reconversion économique : Le cas de Montréal, Paris, L'Harmattan. 